\title{
Analysis of Cluster Interconnection Schemes in 802.15.4 Beacon Enabled Networks
}

\author{
JELENA MIS̆IĆ and RANJITH UDAYSHANKAR \\ Department of Computer Science, University of Manitoba, Winnipeg, Manitoba, \\ Canada
}

\begin{abstract}
In this paper, we consider the interconnection of IEEE 802.15.4 beacon enabled network clusters. We discuss two types of interconnections. One type can be achieved by using the PAN coordinator node as the bridging device and the other type is achieved by using ordinary network nodes as bridge nodes. We discuss design and performance issues of both kinds of interconnections.
\end{abstract}

Keywords Bridging; IEEE 802.15.4; Medium Access Control (MAC); Wireless sensor networks

\section{Introduction}

The recent IEEE 802.15.4 standard [1] for low rate wireless personal area networks supports small, cheap, energy-efficient devices operating on battery power that require little infrastructure to operate [2], [3]. It is considered as enabling technology for home networks and wireless sensor networks. IEEE 802.15.4 networks can appear in star topology where all communications are routed through the PAN coordinator or in peer-to-peer topology where nodes can communicate with each other directly while the PAN coordinator is still needed for cluster management [1]. Networks with peer-to-peer topology have homogeneous nodes with the same initial energy, computational resources, and link capacity, while in star topology the PAN coordinator can have higher energy, computational resources, and potentially higher capacity of inter-coordinator links than ordinary nodes in the cluster.

In the recent period, several evaluations related to the performance of IEEE 802.15.4 networks either in peer to peer or in cluster topology have been conducted and the results have been reported in [4], [5], [6], [7], [8], [9], [10], [11], [12], [13]. The choice of topology for IEEE 802.15.4 standard is still an open question. However, it seems that the choice of topology is an issue of tradeoff between the node simplicity and homogeneity versus the duration of network lifetime. For sensor networks covering large geographic areas it is difficult to replace the sensor's batteries when they are exhausted, and therefore, when nodes close to the sink die the whole network is unavailable. It was shown in [14] that in the homogeneous network case, nodes close to the sink die first since their batteries are exhausted due to excessive packet relaying. The concept of power heterogeneity enhanced with link heterogeneity was further considered in [15] and it was proven that a modest number of nodes with higher power can provide 5-fold increase of network life-time. For this reason we choose cluster with star topology as the basic

Address correspondence to Jelena Mišić, Department of Computer Science, University of Manitoba, Winnipeg, Manitoba, Canada. E-mail: jmisic@cs.umanitoba.ca 
network building block and explore the ways to achieve efficient cluster interconnection in order to implement larger networks.

In this paper, we consider two 802.15.4 clusters operating in beacon enabled, slotted CSMA-CA mode; the clusters will be referred to as the source and sink cluster. The clusters can be interconnected in two ways:

- In Master-Slave (MS) fashion, with the coordinator node of the source cluster acting as the bridge.

- In Slave-Slave (SS)fashion where bridge is ordinary node in both clusters.

The bridge periodically visits the sink cluster in order to deliver the data gathered from the sensor nodes in the source cluster. Bridge visits are made possible by the existence of active and inactive parts of the superframe, i.e., the bridge visits the sink cluster during the inactive period of the source cluster superframe. The bridge delivers its data either by competing with other nodes in the sink cluster using the CSMA-CA access mode, or by using the guaranteed time slots (GTS) allocated by the sink cluster coordinator. Also, standard [1] allows transmissions in the CSMA part of the superframe to be acknowledged giving base for the reliable MAC with re-transmissions or non-acknowledged which has applications in sensor networks where the content of the packet is correlated. In this paper, we discuss and compare the performance of MS and SS based bridges. At this point we do not consider power management algorithms explicitly since we target applications of both Personal Area Networks where reliable MAC is needed and Sensor Networks which can demand either reliable or non-reliable MAC. However, our framework is open for power management algorithms either through control of inactive superframe part or through individual power control for each node.

The rest of the paper is organized as follows. In section II we review the properties of 802.15.4 beacon enabled MAC related to the operation of bridges. In Section III, we present the details of Master-Slave (MS) bridge operation. In Section IV the operation of Slave-Slave (SS) bridge is described. In Section V, we discuss comparative performance issues of MS bridges and SS bridges. Finally, Section VI concludes the paper.

\section{Basic Properties of IEEE Std 802.15.4 MAC}

In beacon enabled networks the PAN coordinator divides its channel time into superframes [1]. Each superframe begins with the transmission of a network beacon, followed by an active portion and an optional inactive portion, as shown in Fig. 1. The coordinator interacts with its PAN during the active portion of the superframe, and may enter a low power mode during the inactive portion. The superframe duration, $\mathrm{SD}$, is equivalent to the duration of the active portion of the superframe, which cannot exceed the beacon interval BI.

All communications in the cluster take place during the active portion of the superframe, the duration of which is referred to as the superframe duration $S D$. The superframe is divided into 16 slots of equal size. Each slot consists of $3 \cdot 2^{S O}$ backoff periods which gives the shortest active superframe duration aBaseSuperframeDuration of 48 backoff periods. In the ISM band, the duration of the backoff period is 10 bytes, giving the maximum data rate of $250 \mathrm{kbps}$. The duration of the active part of the superframe is $S D=$ aBaseSuperframeDuration $\times 2^{S O}$ (expressed in backoff periods), where the parameter $S O=0 \ldots 14$ is known as macSuperframeOrder. The time interval between successive beacons is $B I=$ aBaseSuperframeDuration $* 2^{B O}$, where $B O$ denotes the so-called macBeaconOrder. The duration of the inactive period of the superframe can easily be determined as $I=$ aBaseSuperframeDuration $*\left(2^{B O}-2^{S O}\right)$. While the default access 
mode in the beacon enabled operation is slotted CSMA-CA, some slots may optionally be reserved for certain devices (GTS). Any device can request a GTS (one for uplink and/or one for downlink transmission), but the actual allocation is ultimately the responsibility of the cluster coordinator. The structure of the superframe is shown in Fig. 1.

Data transfers from a node to PAN coordinator are synchronized with beacons as shown in Fig. 2(a) and are done using slotted CSMA-CA access described below. Data transfers from the coordinator are more complex and are first announced by the coordinator which transmits the list of nodes which have the pending downlink packets. The device periodically listens to the network beacon and if a packet is pending, transmits a MAC command requesting the data. MAC command frames are very short.

The PAN coordinator acknowledges the successful reception of the data request by transmitting an acknowledgement frame. After the acknowledgement the node turns on its receivers for a period of aMaxFrameResponseTime which is equal to 1220 symbols and the PAN coordinator has to transmit the pending frame within that period.

Downlink transmission can be achieved without slotted CSMA-CA only if the coordinator's MAC layer can start transmission of the data frame between aTurnaroundTime (12 symbols) and aTurnaroundTime + aUnitBackoffPeriod and there is time remaining in the Contention Access Part for the packet appropriate inter-frame spacing and acknowledgement. If this is not possible transmission is done using slotted CSMA-CA. We consider that downlink transmissions without CSMA-CA mechanism are detrimental for network performance since they will cause additional collisions and we assume that all transmissions will be achieved using slotted CSMA-CA.

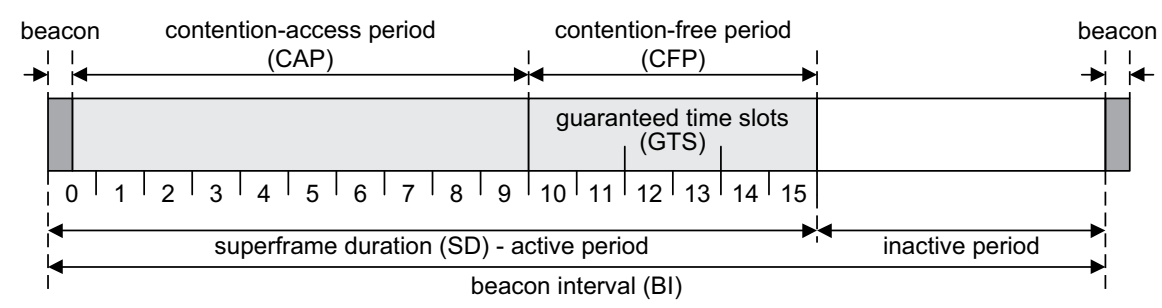

Figure 1. The composition of the superframe (adapted from [1]).

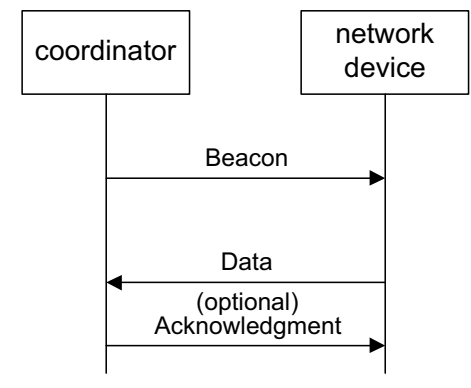

(a) Uplink transmission.

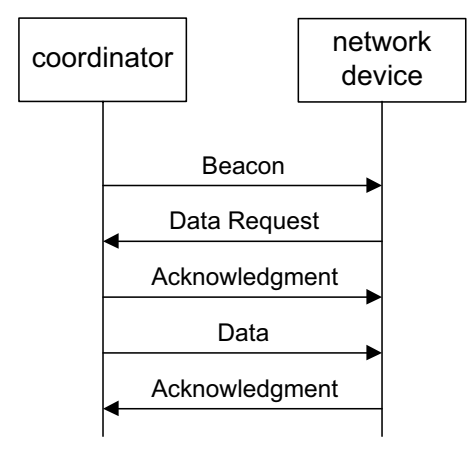

(b) Downlink transmission.

Figure 2. Uplink and downlink data transfers in beacon enabled PAN. 
If the transmission was correctly received within the time limit, the node will acknowledge it. If not, the whole process of announcement through the beacon has to be repeated. The standard allows for informing the device that there are more frames waiting at the PAN coordinator's queue by using the frame pending subfield of the data frame received from the coordinator (a more bit). If more bit is set to 1 , the device still has frames with the coordinator and it has to send a new data request frame in order to retrieve it. The cycle of downlink transmission is shown in Fig. 2(b). According to the standard, maximally 7 devices can be advertized in the beacon. In this work we assume that PAN will advertize nodes in round-robin xfashion in the case if it has more than 7 pending downlink packets.

The active portion of each superframe is divided into equal sized slots; the beacon is transmitted at the beginning of slot 0 , and the contention access period (CAP) of the active portion starts immediately after the beacon. In each slot, the channel access mechanism is contention based, using the CSMA-CA access mechanism (more details are given below). A device must complete all of its contention based transactions within the contention access period (CAP) of the current superframe.

Within the time slots of the active portion of the superframe, the PAN coordinator may reserve slots to allow dedicated access to some devices. These slots are referred to as guaranteed time slots (GTS), and together they comprise the so-called contention-free period (CFP). In this work we do not consider the impact of the GTS, although their presence will clearly decrease the usable bandwidth of the PAN for other devices.

The basic time unit of the MAC protocol is the duration of the so-called backoff period. Access to the channel can occur only at the boundary of the backoff period. The actual duration of the backoff period depends on the frequency band in which the 802.15.4 WPAN is operating. Namely, the standard allows the PAN to use either one of three frequency bands: $868-868.6 \mathrm{MHz}, 902-928 \mathrm{MHz}$, and 2400-2483.5 MHz. In the two lower frequency bands, BPSK modulation is used, giving the data rate of $20 \mathrm{kbps}$ and $40 \mathrm{kbps}$, respectively. Each data bit represents one modulation symbol which is further spread with the chipping sequence. In the third band, the O-QPSK modulation is used before spreading; in this case, four data bits comprise one modulation symbol which is further spread with the 32-bit spreading sequence. Table 1 summarizes the basic timing relationships in the MAC sub-layer. Note that the constants and attributes of the MAC sub-layer, as defined by the standard, are written in italics. Constants have a general prefix of "a", e.g. aUnitBackoffPeriod, while attributes have a general prefix of "mac", e.g. macMinBE.

\subsection{CSMA-CA Algorithm}

This algorithm is comprised of the downlink data transmission, uplink data transmission, and uplink request transmission states. As is the case with other contention-based access control schemes, transmission will be attempted only when the medium is clear, but withheld if there is channel activity, or when contention occurs. The CSMA-CA protocol, shown as a flowchart in Fig. 3, is invoked when a packet is ready to be transmitted. In this algorithm, three variables are maintained for each packet:

1) $N B$ is the number of times the algorithm was required to backoff due to the unavailability of the medium during channel assessment;

2) $C W$ is the contention window, i.e., the number of backoff periods that need to be clear of channel activity before the packet transmission can begin; and 


\section{Table 1}

Timing Structure of the Slotted Mode MAC Protocol. (Note that the Values of Voth $B O$ and $S O$ Must be Less than 15 in the Beacon Enabled Mode.)

\begin{tabular}{|c|c|c|}
\hline Type of time period & Duration & MAC constant \\
\hline Modulation symbol & $\begin{array}{l}1 \text { data bit in } 860 \mathrm{MHz} \text { and } \\
915 \mathrm{MHz} \text { bands, } 4 \text { data } \\
\text { bits in } 2.4 \mathrm{GHz} \text { band }\end{array}$ & N/A \\
\hline Unit backoff period & 20 symbols & aUnitBackoffPeriod \\
\hline $\begin{array}{l}\text { Basic superframe slot } \\
\quad(\mathrm{SO}=0)\end{array}$ & $\begin{array}{l}\text { three unit backoff periods } \\
\text { (60 symbols) }\end{array}$ & aBaseSlotDuration \\
\hline $\begin{array}{l}\text { Basic superframe length } \\
\quad(\mathrm{SO}=0)\end{array}$ & $\begin{array}{l}16 \text { basic superframe slots } \\
\text { (960 symbols) }\end{array}$ & $\begin{array}{l}\text { aBaseSuperframeDuration } \\
=\text { NumSuperframeSlots } \\
\text { aBaseSlotDuration }\end{array}$ \\
\hline $\begin{array}{l}\text { (Extended) superframe } \\
\text { duration SD }\end{array}$ & aBaseSuperframeDuration $\cdot 2^{S O}$ & macSuperframeOrder, SO \\
\hline Beacon interval BI & aBaseSuperframeDuration $\cdot 2^{B O}$ & macBeaconOrder, $\mathrm{BO}$ \\
\hline $\begin{array}{l}\text { Maximal time to wait for } \\
\text { downlink } \\
\text { transmission }\end{array}$ & 1220 symbols & aMaxFrameResponseTime \\
\hline $\begin{array}{l}\text { Rx-to-Tx or Tx-to-Rx } \\
\text { maximum } \\
\text { turnaround time }\end{array}$ & 12 symbols & aTurnaroundTime \\
\hline $\begin{array}{l}\text { Time-out value to wait for } \\
\text { the acknowledgement. }\end{array}$ & 54 symbols & macAckWaitDuration \\
\hline
\end{tabular}

3) $B E$ is the backoff exponent which is related to the number of backoff periods a device should wait before attempting to assess the channel (see below for a detailed explanation).

In step (1), the algorithm begins by setting $N B$ to zero and $C W$ to 2 . If the device operates on battery power (as determined by the attribute macBattLifeExt), BE is set to 2 or to the constant macMinBE, whichever is less; otherwise, it is set to macMinBE (the default value of which is 3 ). The algorithm then locates the boundary of the next backoff period.

In step (2), the algorithm attempts to avoid collisions by generating random waiting time in the range $0 \ldots 2^{B E}-1$ backoff periods. When the wait period is over, the MAC sub-layer needs to perform $C W$ clear channel assessment (CCA) procedures, transmit the frame, and optionally wait for acknowledgment. The time to wait for an acknowledgment, macAckWaitDuration, is equivalent to 54 or 120 symbols, depending on the currently selected physical channel. If the remaining time within the CAP area of the current superframe is sufficiently long to accommodate all of these, the MAC sublayer will proceed with step (3) and perform the first CCA to see whether the medium is idle. If the remaining time is not sufficient, the MAC sublayer will pause until the next superframe.

If the channel is busy, the values of $N B$ and $B E$ are increased by one (but $B E$ cannot exceed macMaxBE, the default value of which is 5), while $C W$ is reset to 2 ; this is step (4) 


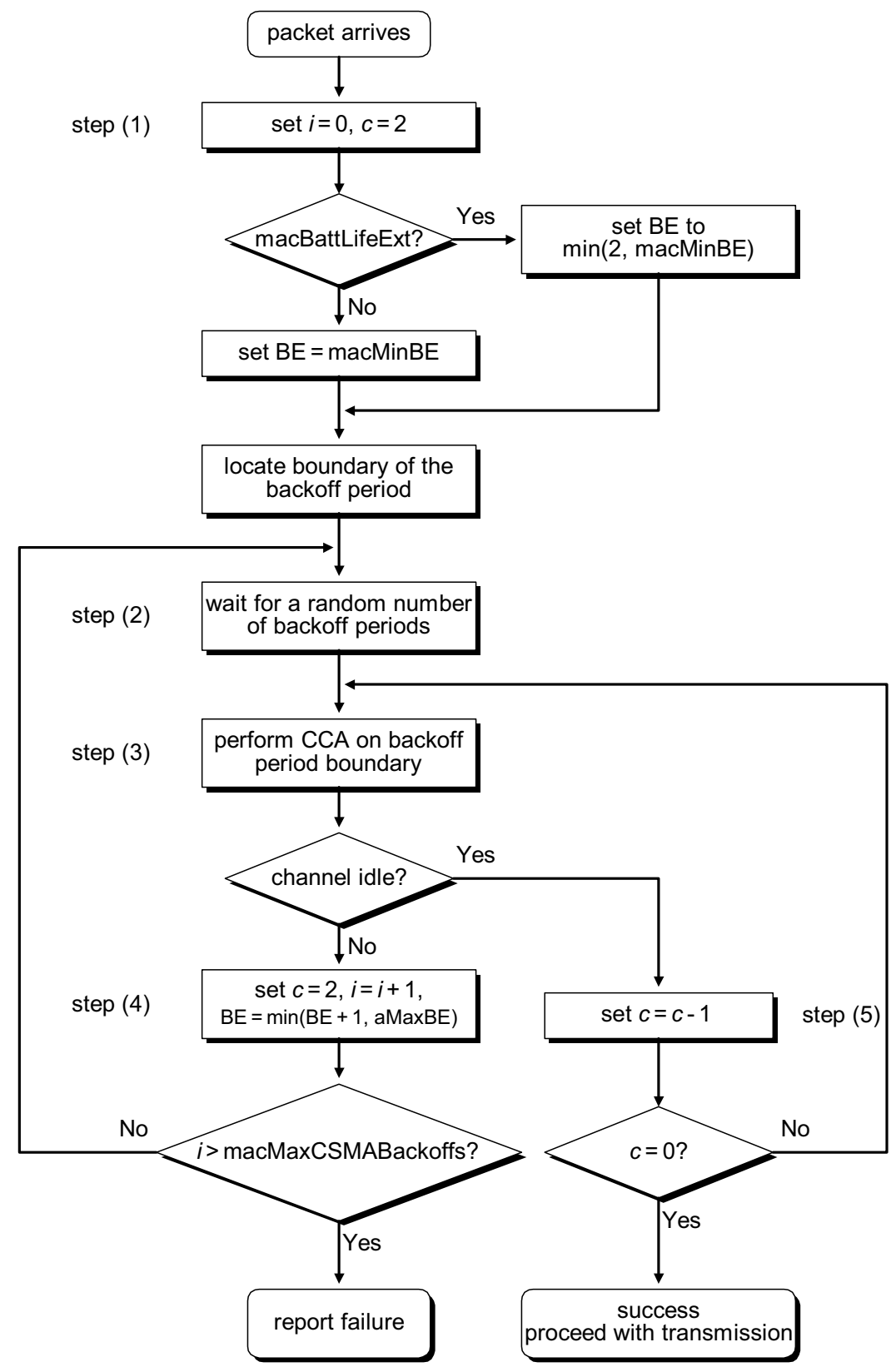

Figure 3. Operation of the slotted CSMA-CA MAC algorithm in the beacon enabled mode (adapted from [1]).

in the flowchart. If the number of retries is below or equal to macMaxCSMABackoffs (the default value of which is 5), the algorithm returns to step (2), otherwise the algorithm terminates with a channel access failure status. Failure will be reported to the higher protocol layers, which can then decide whether to re-attempt the transmission as a new packet or not. 
If the channel is idle, step (5), the value of $C W$ is decreased by one, and the channel is assessed again. When $C W$ becomes zero, the transmission of the packet may begin, provided the remaining number of backoff periods in the current superframe suffices to handle both the packet and the subsequent acknowledgment. If this is not the case, the standard requires that the transmission is deferred until the beginning of the next superframe.

Note that the backoff unit boundaries of every device should be aligned with the superframe slot boundaries of the PAN coordinator, i.e., the start of the first backoff unit of each device is aligned with the start of the beacon transmission. The MAC sub-layer should also ensure that the PHY layer starts all of its transmissions on the boundary of a backoff unit.

\section{Master-Slave Bridging Algorithm}

We consider two interconnected clusters operating in the ISM band around 2.4GHz. Each cluster operates in different frequency sub-band so that inter-cluster interference is avoided (standard prescribes 16 distinct cluster channels). We assume that both clusters operate in beacon enabled CSMA-CA mode and control of their respective cluster (PAN) coordinators. In each cluster, the channel time is divided into superframes which are bounded by beacon transmissions from the coordinator [1]. For clarity, variables pertaining to the source and sink cluster will be labeled with subscripts $s r c$ and $s n k$, respectively, while the variables linked to the bridge will have the subscript bri.

During the inactive portion of the superframe shown in Fig 2, any device may enter a low power mode or perform other functions, including the interconnection function. This facilitates the creation of larger networks through bridging, with the cluster coordinator of the source cluster acting as the bridge. When the active part of the superframe is completed in the source cluster, its cluster coordinator/bridge switches to the sink cluster. The bridge has stored the packet(s) that need to be delivered to the sink cluster coordinator (which acts as the network sink), and it waits for the beacon so that it can deliver its data to the sink cluster coordinator.

In case the bridge has been allocated GTS access, it will wait until its slot arrives and then transmit the data without any backoff countdown; otherwise, it will execute the CSMA-CA transmission procedure just like any other node in sink cluster. In the latter case, should the bridge be unable to transmit its data when the (active portion of the) superframe in the sink cluster ends, it will freeze its backoff counter and leave the sink cluster. The bridge will resume the backoff countdown upon returning to the sink cluster for the next superframe. Also, if the bridge's buffer becomes empty before the end of the sink's active superframe part, the bridge will immediately return to the source cluster and wait for the time to transmit the beacon denoting the beginning of the next superframe, and the source cluster continues to operate. The bridge operation in both access modes is presented in Fig. 4; in the discussions that follow, we will refer to those modes as the CSMA-CA and GTS mode, respectively.

We will assume that all the traffic from the source cluster occurs in the uplink direction, and that the bridge actually delivers it to the sink cluster coordinator. The sink cluster has some local traffic as well. (In more complex networks, the sink cluster may contain several bridges, each with its own "source" cluster.) This is a reasonable assumption in sensor networks, where the most, if not all, of the traffic will be directed toward the network sink. All ordinary nodes in either cluster use CSMA-CA access mode. 
(a)

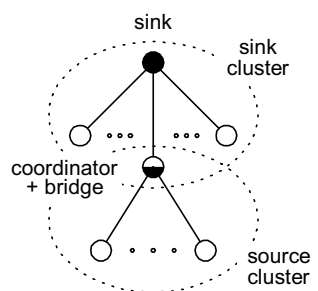

(b)

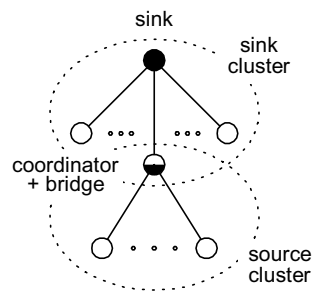

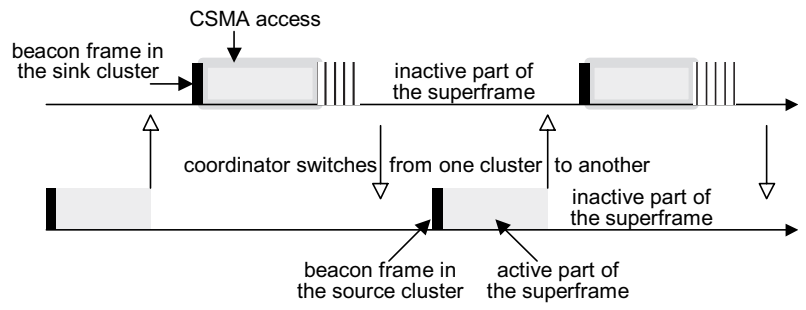

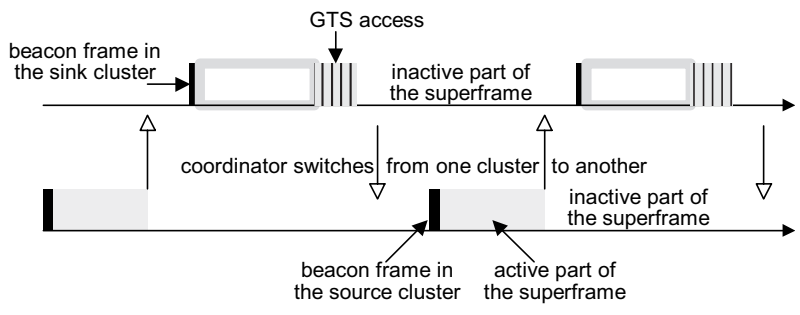

Figure 4. Bridge switching in CSMA-CA and GTS mode, respectively.

\subsection{Queuing Model of MS Bridge Exchange}

Let us now consider the source cluster and calculate the amount of traffic that reaches the sink cluster. The source cluster contains $n$ ordinary sensor nodes which have the packet arrival rate of $\lambda_{i}$ during inactive and active parts of the superframe. Coordinator in the source cluster also functions as the bridge. During the active part of the superframe nodes send packets to the bridge. However, not all generated packets make it to the transmission medium because of packet blocking at the source nodes. Namely, the ordinary sensor node is assumed to have finite input buffer with the capacity of $L$ packets; once the buffer is full, the packets will be simply dropped. This poses little problems in sensing applications as redundant information is available from other nodes. The coordinator/bridge has a finite buffer as well; this buffer has the capacity of $L_{b r i}$ packets. In case this buffer is full, new packets will not be admitted and the coordinator/bridge will not send the acknowledgment. Let us denote the blocking probabilities at ordinary nodes in the source cluster and at the bridge as $P b_{s r c}$ and $P b_{b r i}$, respectively.

1) Non-acknowledged Transfer. In the case of non-acknowledged transmission, the cluster coordinator does not acknowledge the packets which are successfully received and stored in its buffer. Without acknowledgements, packets transmitted towards the bridge which are lost due to the noise at the physical layer, collisions or blocking will not be re-transmitted.

The amount of traffic admitted in the source cluster is $n \lambda_{i}\left(1-P b_{s r c}\right)$ and the total packet arrival rate offered to the bridge is $\lambda_{b r i}=n \lambda_{i}\left(1-P b_{s r c}\right) \gamma_{s r c} \delta_{s r c}$, where $\gamma_{s r c}$ denotes probability that no collision has occurred for a particular packet in the source cluster and $\delta_{s r c}$ denotes the probability that the packet is not corrupted by the noise. Given the bit error rate of the physical medium and the total packet length, $\delta_{s r c}$ can be calculated as the probability that none of the bits in the packet is corrupted by the noise. The graphical 
representation of the queuing, blocking, and bridging between the two clusters is shown in Fig. 5(a).

2) Acknowledged Transfer. The purpose of the acknowledged transfer is to achieve reliable packet transfer at the MAC layer. All successful packet transmissions which are received by the respective cluster coordinator and placed in its buffer are acknowledged. However, when the incoming packet reaches the coordinator/bridge when its buffer is full it will not send the acknowledgment even if the transmission was successful. The lack of acknowledgment may also be due to a collision or noise at the physical layer. If the acknowledgment is not received within the time prescribed by the standard [1], the sending node will repeat the transmission. In our model, the ordinary node will repeat the packet transmission until it receives the acknowledgement. (The standard prescribes

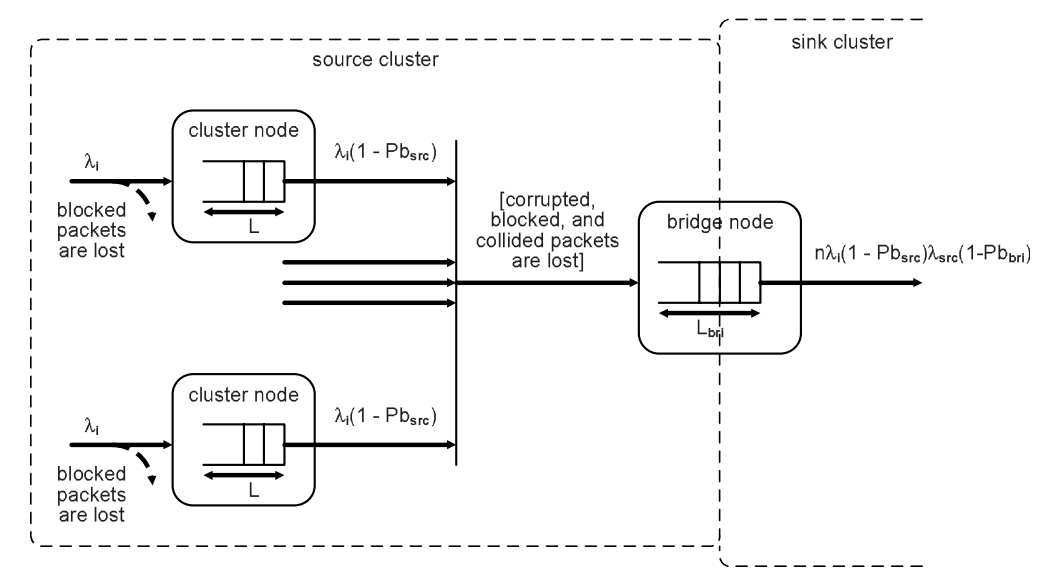

(a) Non-acknowledged transfer.

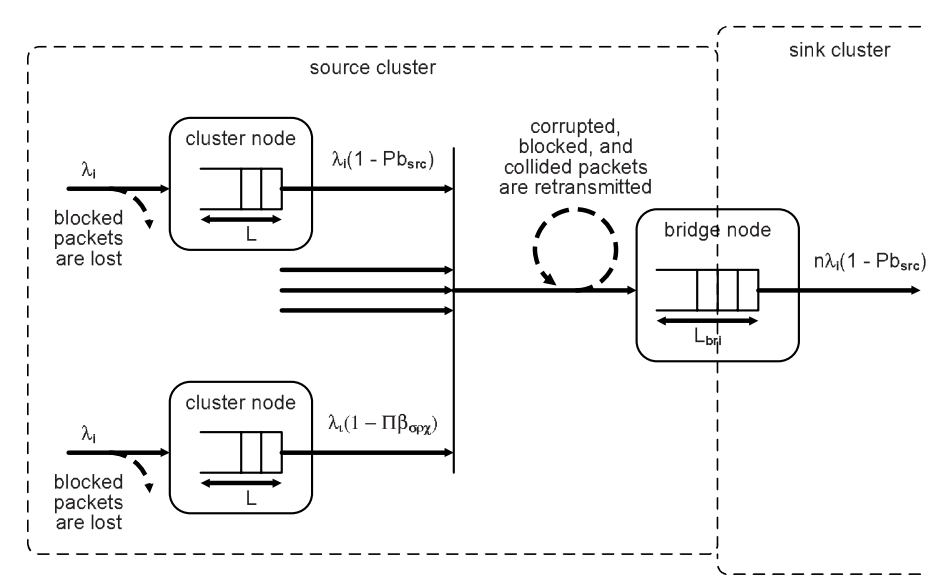

(b) Acknowledged transfer.

Figure 5. Queuing model of the bridging process between source and sink cluster. 
maximum number of transmission re-tries but in that case the final reliability of transmission has to be achieved at higher protocol layers which is equivalent to our approach.)

In this case, we can say that traffic blocked by the bridge "stays" in the network, and contributes to an increase in traffic, as well as the number of collisions, in the source cluster. The graphical representation of the queuing, blocking, and bridging between the two clusters is shown in Fig. 5(b).

The amount of traffic admitted in the source cluster is $n \lambda_{i}\left(1-P b_{s r c}\right)$. Since the transfer is reliable, the total arrival rate offered to the bridge satisfies the following equality $n \lambda_{i}\left(1-P b_{s r c}\right)=\lambda_{b r i}\left(1-P b_{b r i}\right)$, which gives the offered bridge packet arrival rate as

$$
\lambda_{b r i}=\frac{n \lambda_{i}\left(1-P b_{s r c}\right)}{1-P b_{b r i}}
$$

Since the number of nodes $n$ is relatively large and events of packet blocking by the bridge, collisions and corruptions by noise are non-correlated we will assume that packet arrival process to the bridge is Poisson with average rate $\lambda_{b r i}$.

\section{Slave-Slave Bridging Algorithm}

Slave-Slave bridge is an ordinary node in both the source and sink cluster. It visits both clusters in time-division basis as shown in Fig. 6.

Upon leaving one cluster, bridge has to wait for the beacon of new cluster in order to start communication. This synchronization time is also shown in Fig. 7 where $t$ indicates the duration of the superframe and points 1 and 2 indicate the start and end of the bridge's synchronization time.

However, the SS bridge operation is more complex than the MS bridge operation. The reason for this is complex downlink communication where each downlink packet has first to be advertized in the beacon, requested by the bridge node by sending a request packet, the request has to be acknowledged by the coordinator and finally the downlink transmission can commence. The request packet is sent in CSMA-CA mode and can collide with other uplink data packets. The downlink data packet is also sent in the CSMA-CA mode and can collide with the other packets. Standard also prescribes that the downlink transmission has to be completed within 61 backoff periods, and if it comes later due to many backoff attempts it will not be acknowledged by the bridge. The

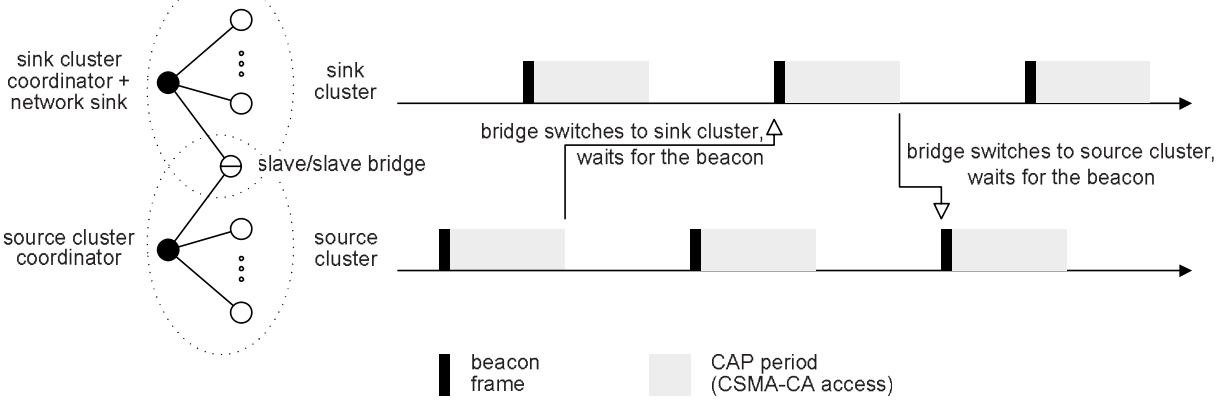

Figure 6. Bridge switching between the source and sink cluster. 


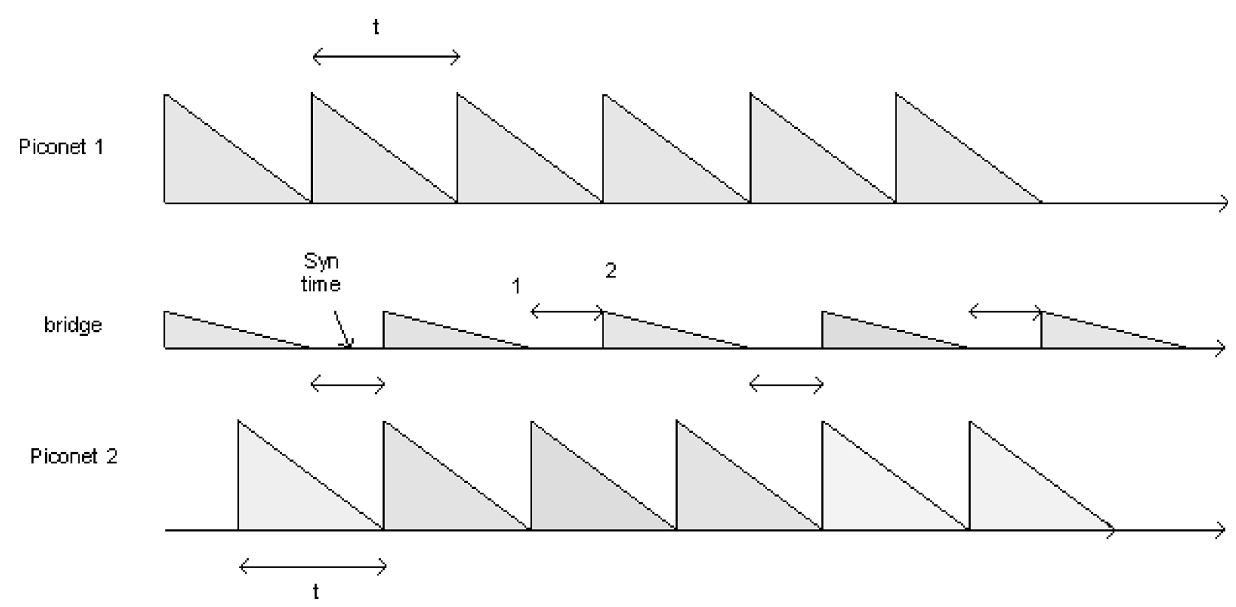

Figure 7. Timing of the SS bridge's presence in source and and sink cluster.

diagram which shows interaction among all the nodes involved in the operation of the source and sink cluster is shown in Fig. 8. Note that this figure indicates operations in active superframe parts from Figs. 6 and 4. As mentioned in Section III, clusters operate in different frequency bands and inter-cluster interference is not present.

From the discussions presented above, the following states can be identified for the source or sink PAN coordinator node:

1) The source or sink coordinator may be transmitting the beacon.

2) The source coordinator may be listening to its nodes and receiving data packets from ordinary nodes or request packets from the bridge node. The sink coordinator only receives data packets from the bridge and its local nodes.

3) The source coordinator may be transmitting the downlink data packet as a result of previously received request packet. As soon as the downlink transmission is finished the source coordinator switches to the listening mode.

Similarly, ordinary or bridge node in the source cluster can be in one of the following states:

1) The ordinary node may be transmitting an uplink data packet.

2) The bridge node may be transmitting an uplink request packet.

3) The bridge node may be in an uplink request synchronization state, which is a virtual state that lasts from the moment of the new downlink packet arrival at the coordinator (or the failure of the previous downlink reception) up to the beginning of the CSMA-CA procedure for the uplink request. Note that the arrivals of the downlink packets at the coordinator follow the Poisson process, whereas the corresponding announcements in the beacon (from which the target node finds out about those packets) do not.

4) The bridge node may be waiting for a downlink packet.

5) The ordinary or bridge node may also be in an idle state, without any downlink or uplink transmission pending or in progress.

In the sink cluster, the ordinary or bridge node may be transmitting uplink data packets to the coordinator. 


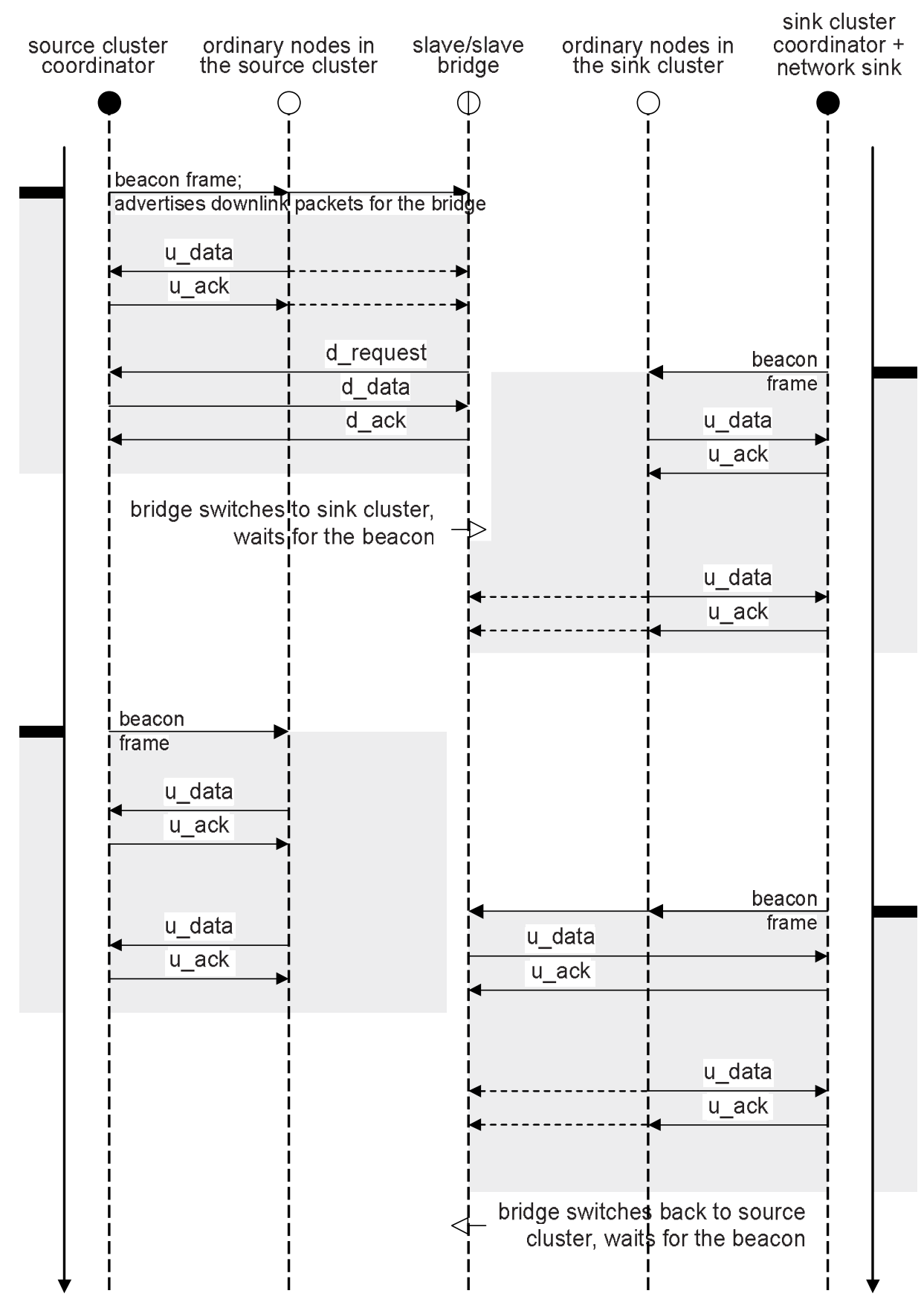

Figure 8. Interactions between the SS bridge, coordinator and ordinary nodes in source and sink cluster.

\subsection{Queuing Model of SS Bridge Exchange}

Let us now consider the source WPAN cluster and calculate the amount of traffic that reaches the sink WPAN cluster. The source WPAN cluster contains $n$ ordinary sensor 
nodes which have the packet arrival rate of $\lambda_{i}$ packets per second during inactive and active time of the superframe. During the active period the superframe nodes send packets to the coordinator and further route the packets to the bridge during its stay. However, not all generated packets make it to the transmission medium because of packet blocking at the source nodes which we discussed in the case of MS bridge. Moreover, the coordinator and the bridge also have a finite buffer; these buffers have the capacity of $L_{c}$ and $L_{b}$ packets. If the buffer is full, a new packet will not be admitted and the node (coordinator or the bridge) will not send the acknowledgment. Let us denote the blocking probabilities at ordinary nodes in the source cluster, the coordinator and at the bridge are $P b_{s r c}, P b_{c}$ and $P b_{b}$, respectively.

1) Non-acknowledged Transfer. In the case of non-acknowledged transmission, the WPAN cluster coordinator does not acknowledge the packets which are successfully received and stored in its buffer. Without acknowledgment, uplink packets which are lost due to the noise at the physical layer, collisions, or blocking will not be re-transmitted. However, request packets sent from the bridge as a result of the coordinator's advertizement of downlink packets have to be acknowledged.

The amount of traffic admitted in the source WPAN cluster is $n \lambda_{i}\left(1-P b_{s r c}\right)$ and the successful packet arrival rate offered to the coordinator is $\lambda_{c}=n \lambda_{i}\left(1-P b_{s r c}\right) \gamma_{s r c} \delta_{s r c}$, where $\gamma_{s r c}$ denotes probability that no collision has occurred for a particular packet in the source cluster and $\delta_{s r c}$ denotes the probability that the packet is not corrupted by the noise. Further, the successful packet arrival rate offered to the bridge is $\lambda_{b}=\lambda_{c}(1-$ $\left.P b_{c}\right) \gamma_{c} \delta_{s r c}$, where $\gamma_{c}$ denotes probability that no collision has occurred for particular packet from coordinator in source cluster.

Given the bit error rate of the physical medium and the total packet length, $\delta_{s r c}$ is calculated as the probability that none of the bits in the packet is corrupted by the noise. The graphical representation of the queuing, blocking, and bridging between the two WPAN clusters is shown in Fig. 9.

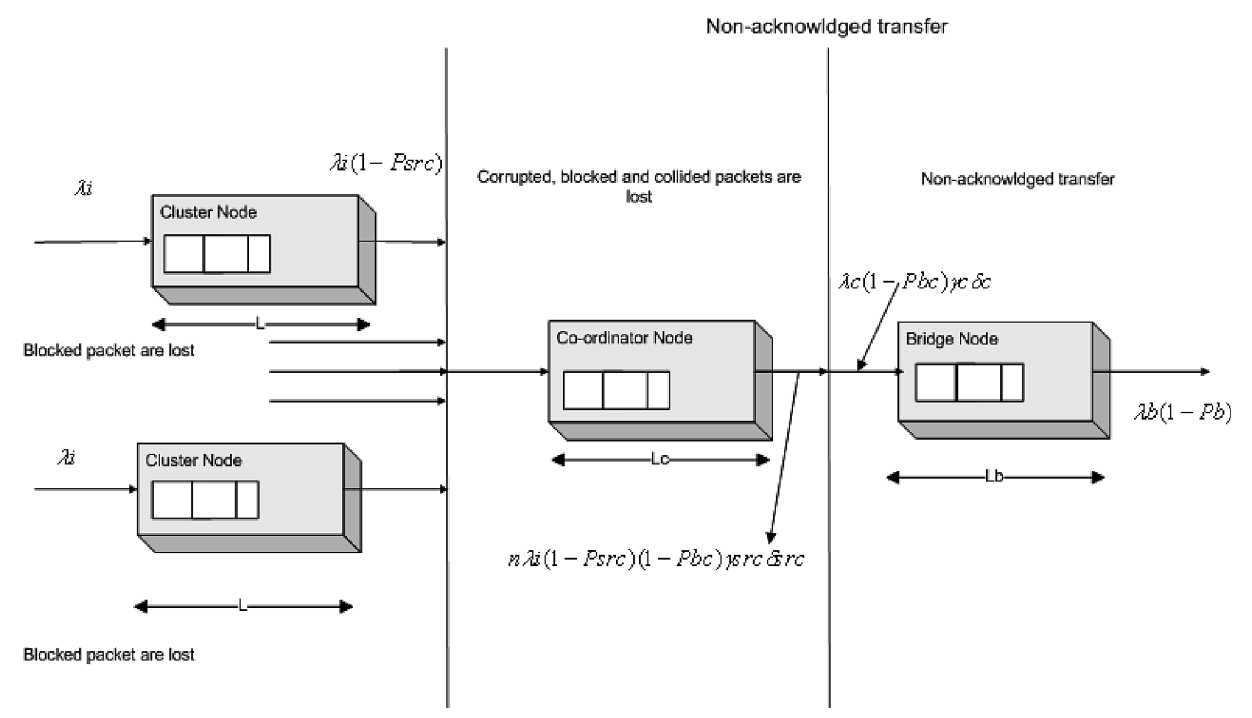

Figure 9. Queuing model of the SS bridging process without packet acknowledgments. 


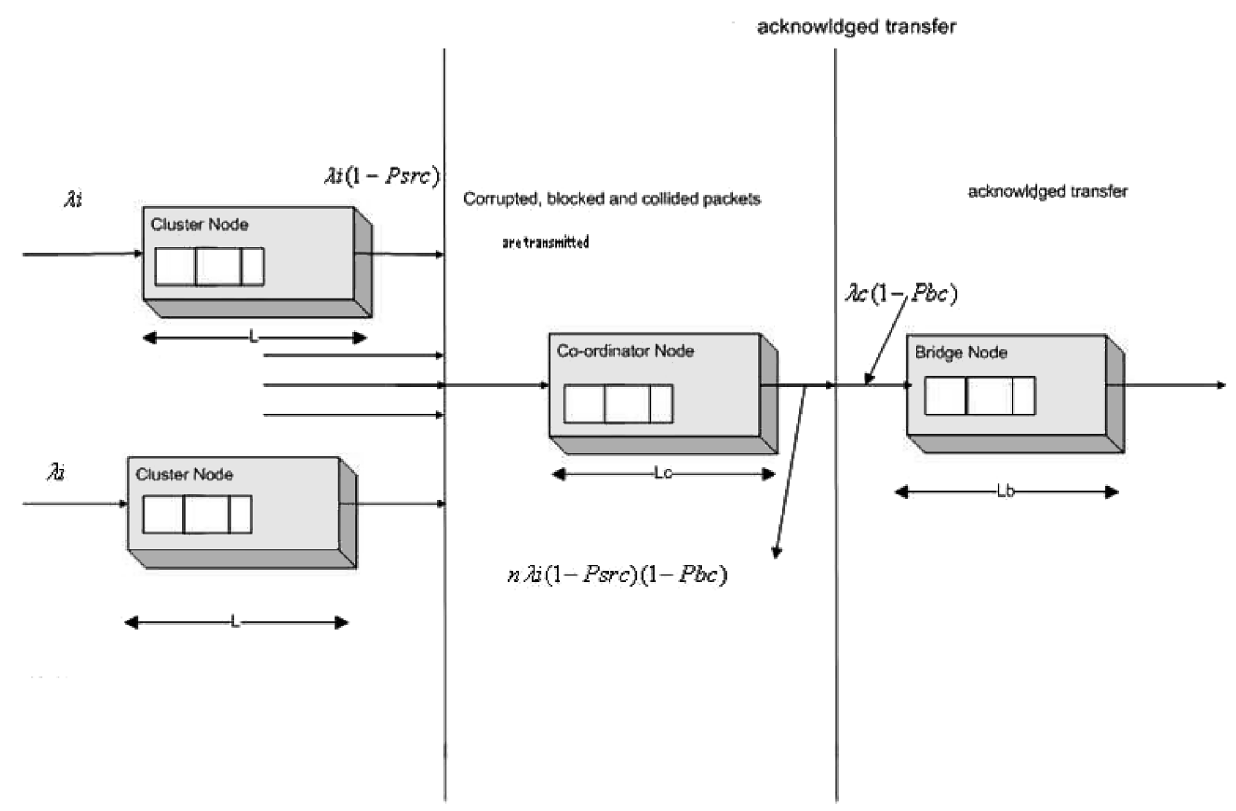

Figure 10. Queuing model of the SS bridging process with reliable packet transmission.

2) Acknowledged Transfer. Acknowledged transfer within 802.15 .4 beacon enabled MAC was discussed in Section III-A.2 and in this Section we only discuss its implications on the operation of SS bridge. The queuing model of bridging between the two WPAN clusters using the SS bridge is shown in Fig. 10.

Since the transfer is reliable, the total data arrival rate offered to the coordinator satisfies the following equality: $n \lambda_{i}\left(1-P b_{s r c}\right)=\lambda_{c}\left(1-P b_{c}\right)$, which gives the offered data packet arrival rate to the coordinator as

$$
\lambda_{c}=\frac{n \lambda_{i}\left(1-P b_{s r c}\right)}{\left(1-P b_{c}\right)}
$$

Similarly, the offered data packet arrival rate to the bridge satisfies the following equality $\lambda_{c}=\lambda_{b}\left(1-P b_{b}\right)$, which gives the offered bridge packet arrival rate as

$$
\lambda_{b}=\frac{n \lambda_{i}\left(1-P b_{s r c}\right)}{\left(1-P b_{c}\right)\left(1-P b_{b}\right)}
$$

Since the number of nodes $\mathrm{n}$ is relatively large and the events of packet blocking by the bridge, collisions, and corruptions by noise are non-correlated we will assume that the packet arrival process to the coordinator is Poisson with average rate $\lambda_{c}$.

\section{Comparative Performance Evaluation}

In this section we compare the performance of MS and SS bridge under CSMA-CA acknowledged bridge access. In the evaluation environment built using the Artifex simulation engine by RSoft Design, Inc. [16] network consists of one source and sink cluster. 
Clusters operate on different channels in the ISM band with raw data rate of $250 \mathrm{kbps}$, which means that aUnitBackoffPeriod has 10 bytes and aBaseSlotDuration has 30 bytes, and bit error rate of $B E R=10^{-4}$. Both the source and the sink cluster had $n$ ordinary nodes, with $n$ varying between 5 and 30 . The packet arrival rate to each ordinary node was varied between 30 packets per minute and 3 packets per second for MS bridge. For the SS bridge packet arrival rate was varied between 80 packets per minute to 280 packets per minute.

Data packet size was fixed at 3 backoff periods and request packet size was 2 backoff periods. Ordinary nodes had buffers that can hold $L=3$ packets, while the bridge buffer capacity was $L_{b r i}=6$ packets.

The superframe size in both clusters was controlled with $S O=0, B O=1$; as the value of aNumSuperframeSlots is 16, the aBaseSuperframeDuration is exactly 480 bytes. The minimum and maximum values of the backoff exponent, macMinBE and $a M a x B E$, were set to three and five, respectively, while the maximum number of backoff attempts was five.

For both MS and SS bridge, the bridge residence time in the source and sink cluster was equal to the active superframe time (48 backoff periods). During residence in the sink cluster the bridge was trying to deliver as many packets as possible. If the bridge's buffer was emptied before the end of the superframe, the bridge has returned to the source cluster. If the bridge was in the process of backoff countdown when the end of the active superframe part occurred, it has frozen the backoff counter and resumed it upon the next visit to the sink cluster.

Figure 11 shows the probability that the medium is idle at first Clear Channel Assessment (CCA) for source nodes, sink nodes, and bridge. By comparing Figs. 11(a) and 11(d) we observe that the nodes in the source cluster will observe less activity on first CCA with the presence of SS bridge. Therefore, the source cluster will enter saturation condition where this probability is low (around 0.4) and flat later when it operates with the SS bridge. This is due to the fact that a large portion of packets in the source cluster with the SS bridge are request packets from the bridge in which the backoff countdown is started immediately after the beacon with the backoff window equal to 8 . Therefore, many request packets from the bridge will choose small backoff value like 0 or 1 and pass the first CCA successfully (first two backoff periods after the beacon are idle). By the same token, the SS bridge will start backoff countdown immediately after joining the sink cluster and sense the idle channel if it gets small backoff value. Similar reasons explain Fig. 12 also. Milder probability that second CCA is successful is just a result of the fact that the bridge's request packets will obtain small backoff values and test the medium in the second or third backoff period after the beacon. Also, when the bridge returns to the sink cluster after being emptied in the previous visit, it will start the backoff count immediately after the beacon and very likely sense that the medium is idle after the beacon. However, the price for a somewhat unrealistic feeling about the activity on the medium in the source cluster is the high level of collisions which is shown in Fig. 13. Indeed, many packets from the bridge which pass the first and second CCAs. However, according to the standard, data packets from the previous superframe which did not have enough room to conduct two CCAs, send the packet and receive the acknowledgement, will have to wait for the next beacon, conduct two CCAs, and transmit. Therefore, request packets from the SS bridge will collide with many delayed packets from the previous superframe which results in the worse success probability for the source cluster with SS bridge than with MS bridge. Large number of collisions of request packets will in turn result in repeated advertisements of downlink transmissions in the beacon and flow of downlink packets to the bridge will slow down until it finally stops. Therefore, relatively good success probabilities for SS bridge in the sink cluster are a result of very few packets which are transferred to the sink cluster and transmitted successfully due to lower congestion in the medium. All these observations are 

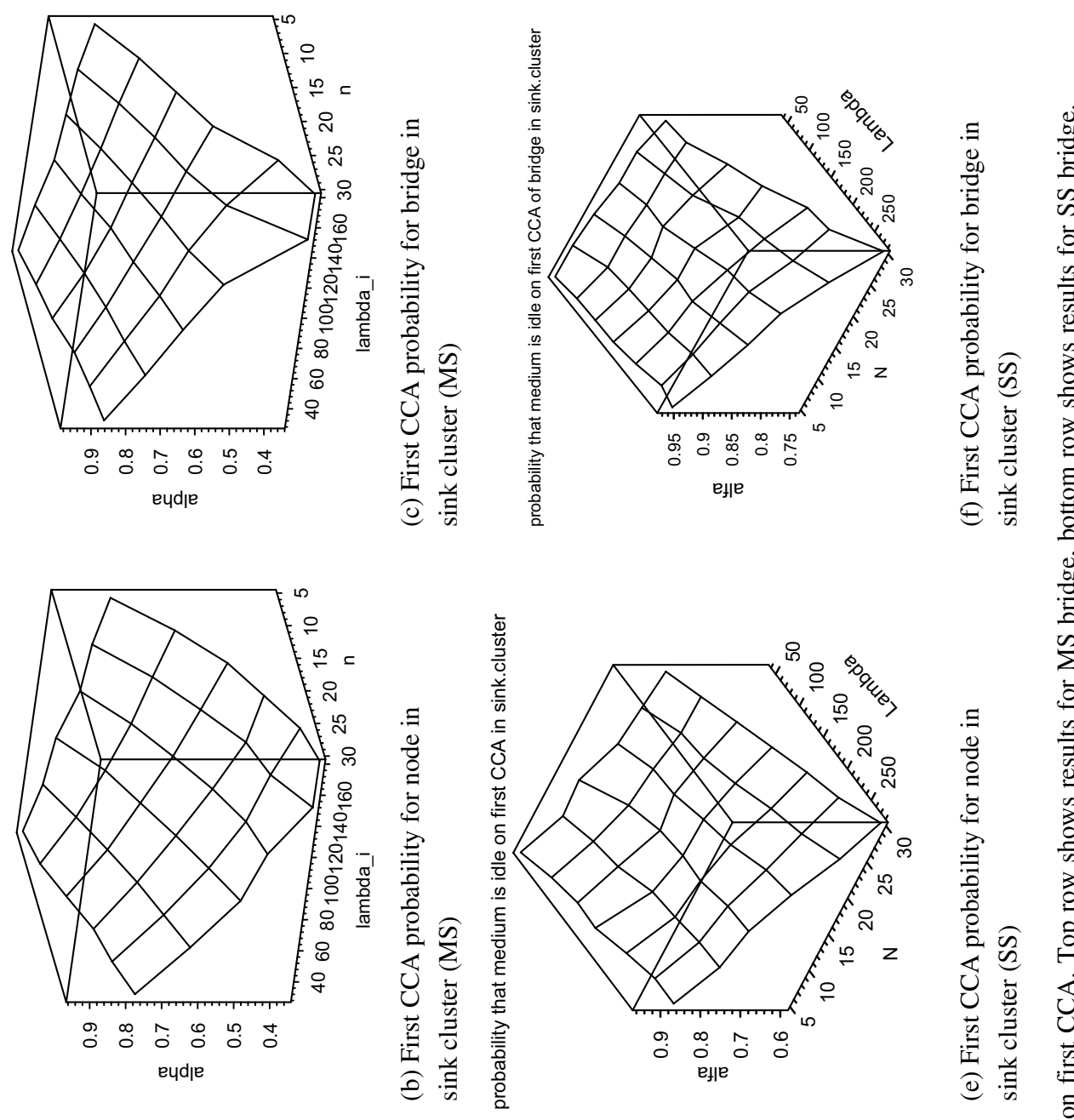

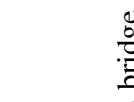
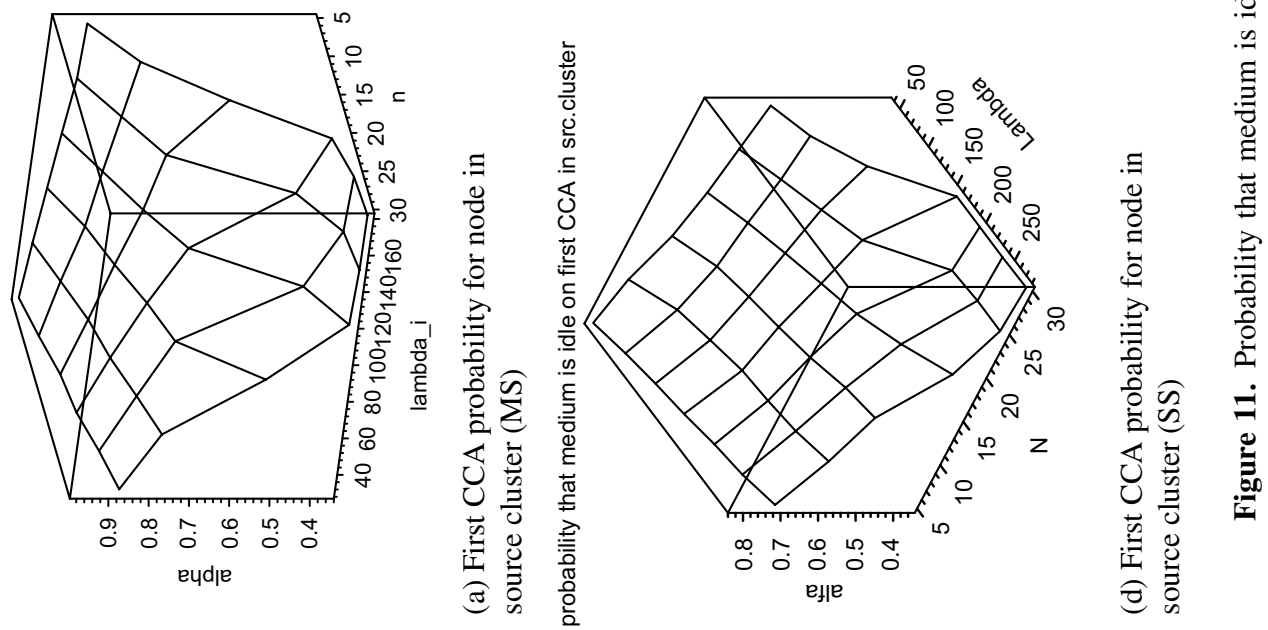

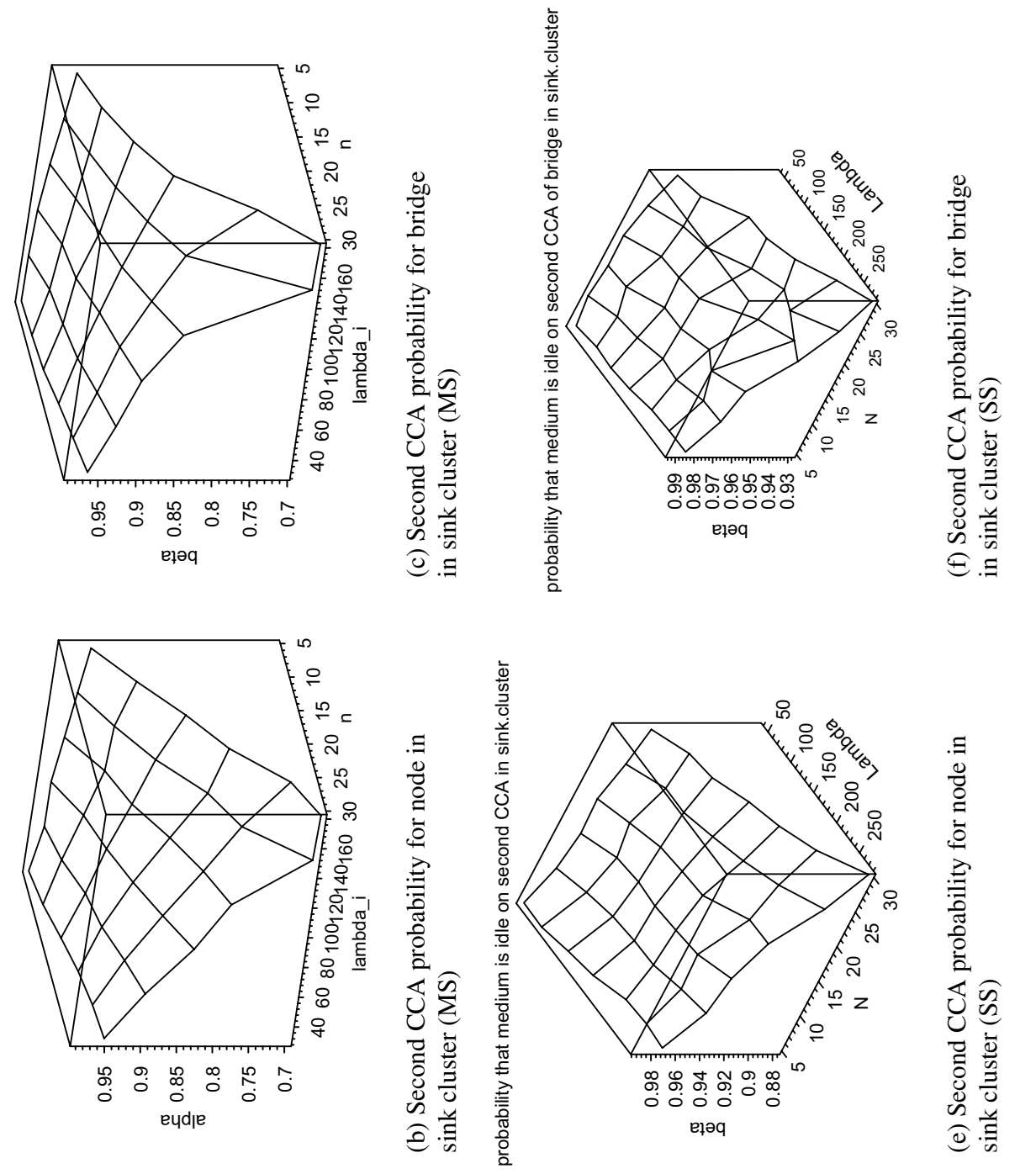

0
0
0
0
0
0
0
0
0
0
0
0
0
0
0
0
0
0
0
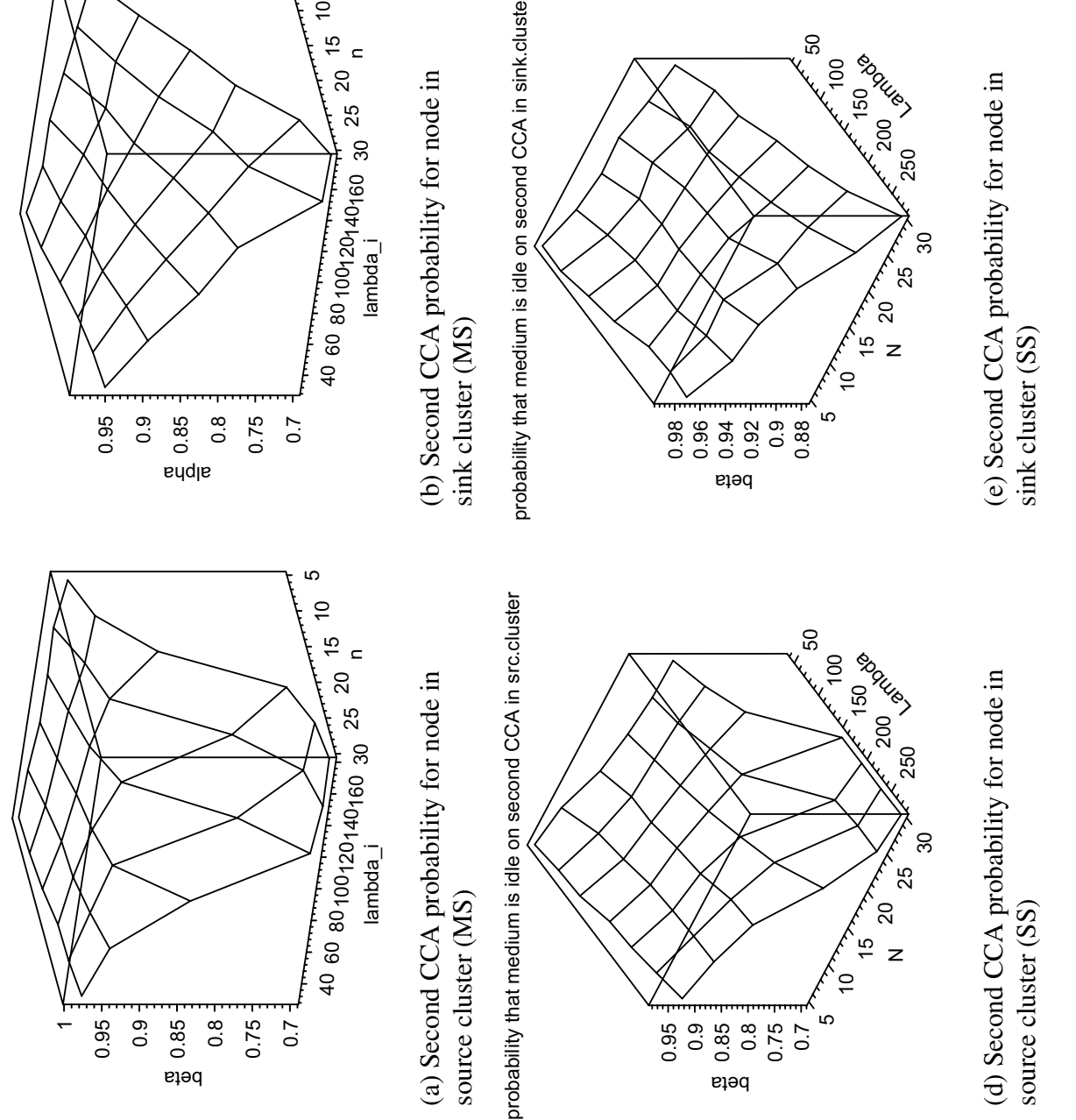

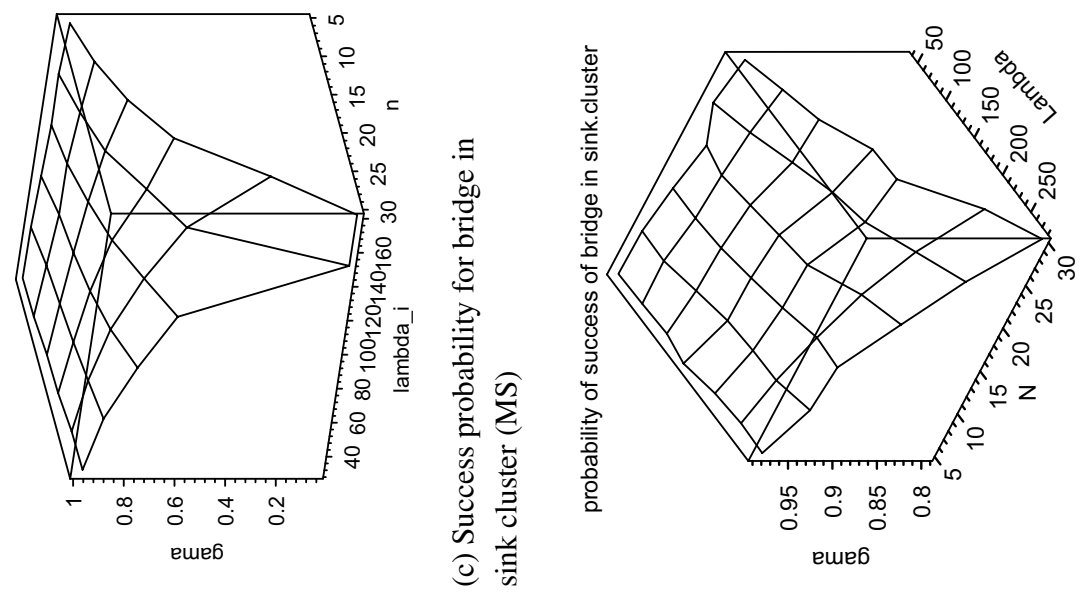

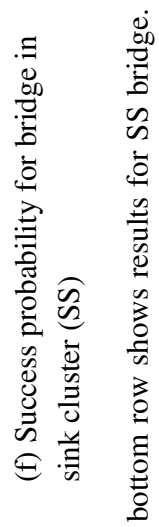
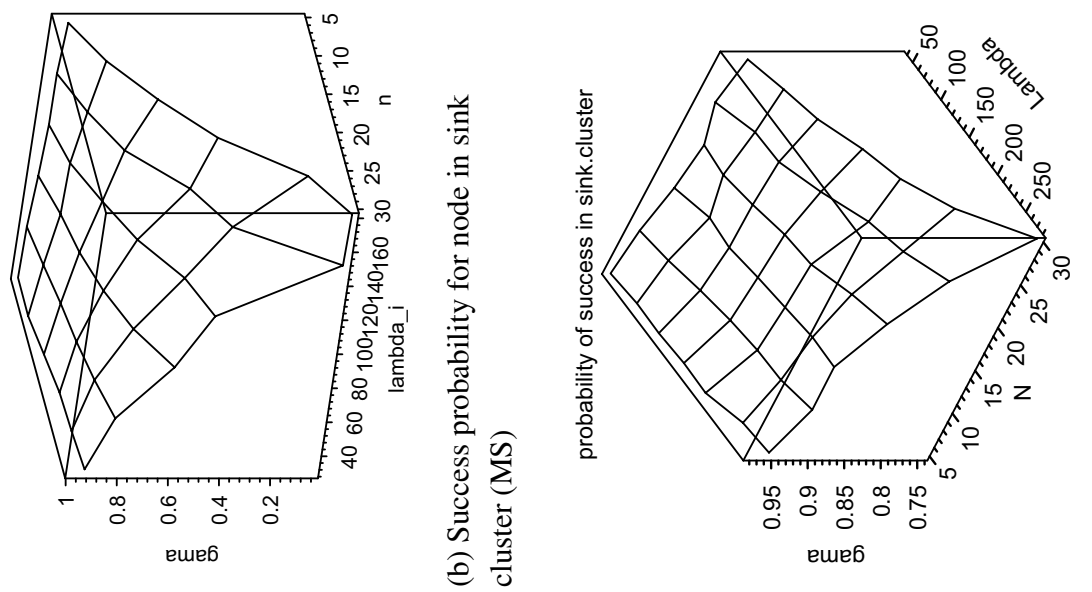

อ

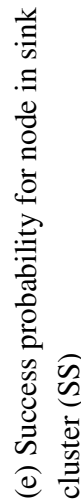
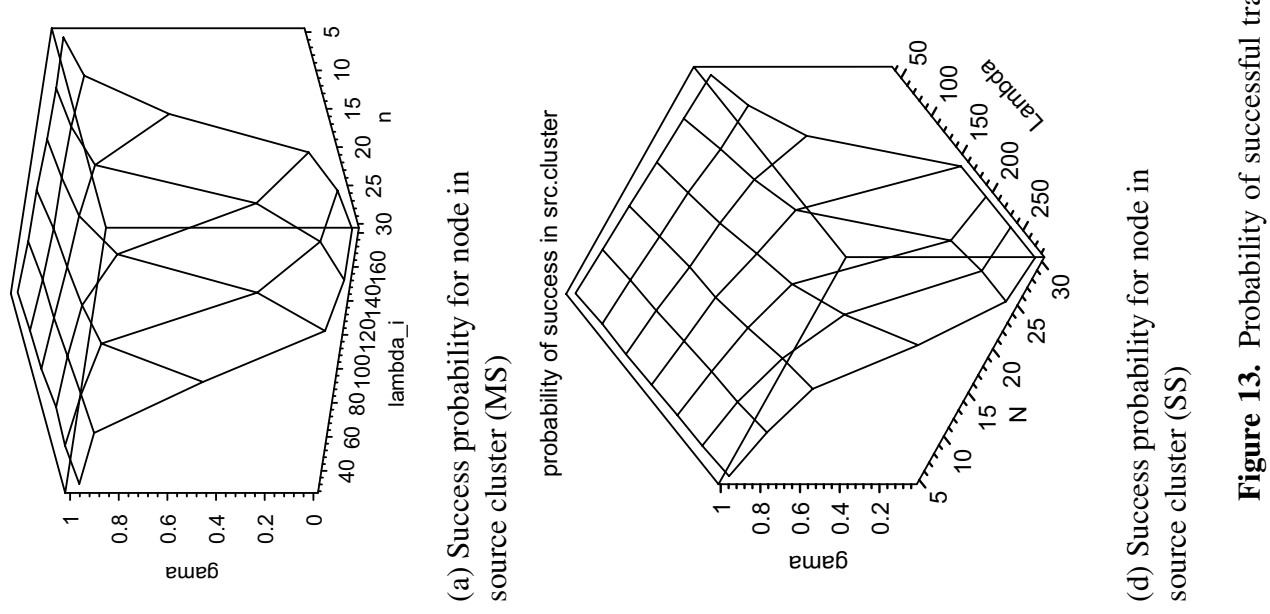

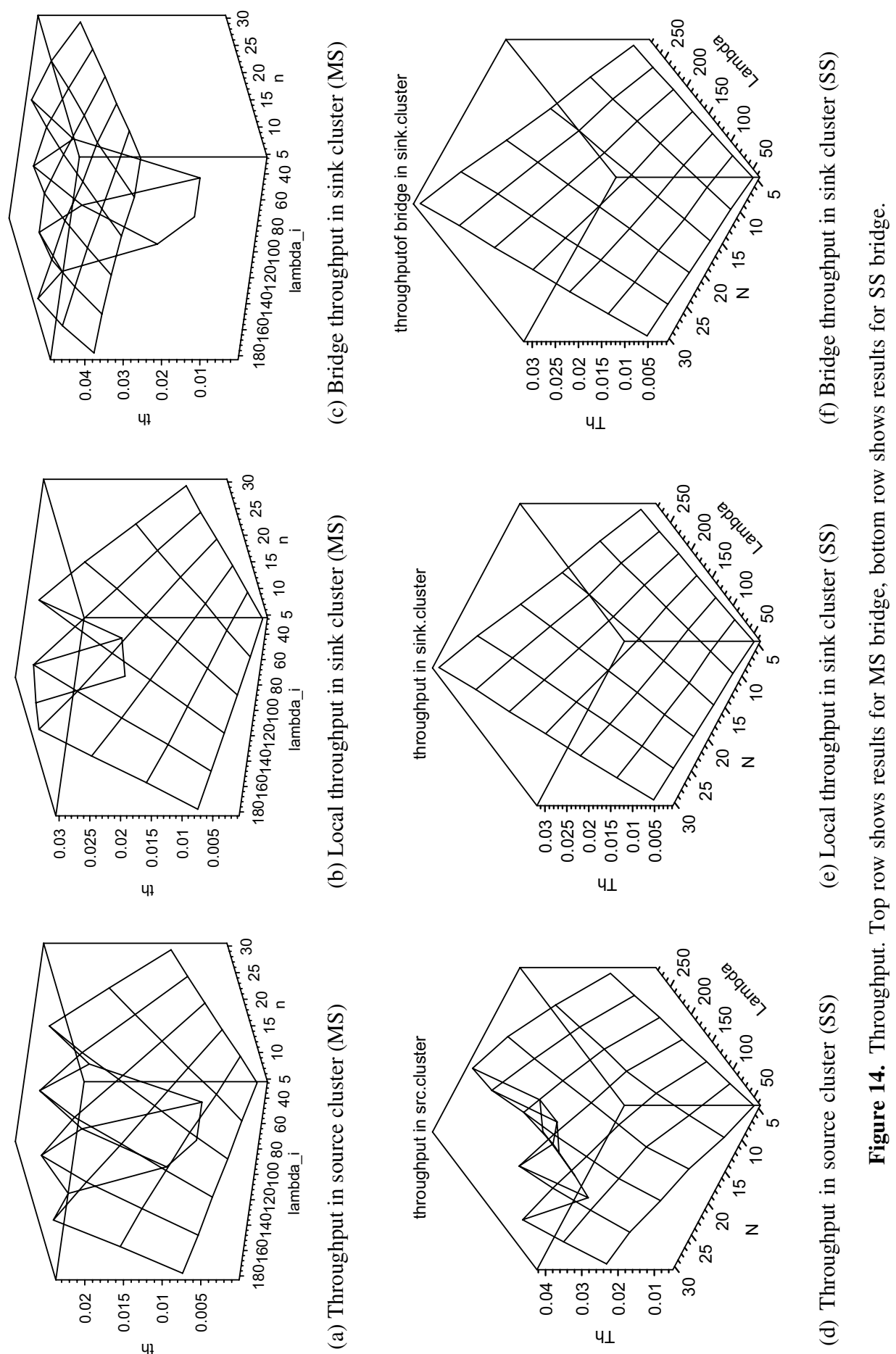
nicely confirmed with throughput values shown in Fig. 14. By comparing Figs. 14(a) and 14(d) we see that throughput in the source cluster reaches a higher maximum value for SS bridge but this is just due to the throughput of the request packets. Bridge throughput in sink cluster is actually higher for MS bridge as shown in Figs. 14(c) and 14(f).

\section{Conclusion}

In this paper we have described design and performance issues of cluster interconnection for beacon enabled 802.15.4 clusters. Our discussion shows that there are pros and cons for both approaches. SS bridge removes the task of bridging from the WPAN coordinator but it generates more traffic in the source cluster. MS bridge efficiently uses the inactive superframe period where all nodes sleep and utilizes uplink data transmissions, but it becomes a single point of failure and target for security attacks.

\section{Acknowledgement}

The authors are grateful to Jun Fung for her help with simulations.

\section{About the Authors}

Jelena Mišić received her Ph.D. in Computer Engineering from the University of Belgrade, Yugoslavia, in 1993. She is currently Associate Professor of Computer Science at the University of Manitoba in Winnipeg, Manitoba, Canada. Her current research interests include wireless networks and security in websites. She is a member of ACM and IEEE.

Ranjith Udayshankar has obtained his M.Sc. degree in Computer Science from the University of Manitoba, Winnipeg, Manitoba, Canada, in the area of performance of wireless networks. He is currently working in Canadian industry.

\section{References}

1. "Standard for part 15.4: Wireless MAC and PHY specifications for low rate WPAN," IEEE, New York, NY, IEEE Std 802.15.4, Oct. 2003.

2. J. A. Gutiérrez, E. H. Callaway, Jr., and R. L. Barrett, Jr., Low-Rate Wireless Personal Area Networks. New York, NY: IEEE Press, 2004.

3. E. H. Callaway, Jr., Wireless Sensor Networks, Architecture and Protocols. Boca Raton, FL: Auerbach Publications, 2004.

4. G. Lu, B. Krishnamachari, and C. Raghavendra, "Performance evaluation of the IEEE 802.15.4 MAC for low-rate low-power wireless networks," in Proc. Workshop on Energy-Efficient Wireless Communications and Networks EWCN'04, Phoenix, AZ, Apr. 2004.

5. J.-S. Lee, “An experiment on performance study of IEEE 802.15.4 wireless networks," in 10th IEEE Conference on Emerging Technologies and Factory Automation ETFA 2005, vol. 2, Catania, Italy, Sept. 2005, pp. 451-458.

6. B. Bougard, F. Catthoor, D. C. Daly, A. Chandrakasan, and W. Dehaene, "Energy efficiency of the IEEE 802.15.4 standard in dense wireless microsensor networks: Modeling and improvement perspectives," in DATE '05: Proceedings of the conference on Design, Automation and Test in Europe, vol. 1, Munich, Germany, Mar. 2005, pp. 196-201.

7. I. Howitt, R. Neto, J. Wang, and J. M. Conrad, "Extended energy model for the low rate WPAN," in Proc. IEEE Int. Conf. on Mobile Ad-hoc and Sensor Systems MASS2005, Washington, DC, Nov. 2005, pp. 315-322. 
8. T. H. Kim and S. Choi, "Priority-based delay mitigation for event-monitoring IEEE 802.15.4 LR-WPANs," IEEE Communication Letters, vol. 10, no. 3, pp. 213-215, Mar. 2006.

9. N. F. Timmons and W. G. Scanlon, "Analysis of the performance of IEEE 802.15 .4 for medical sensor body area networking," in First Annual IEEE Communications Society Conference on Sensor and Ad Hoc Communications and Networks SECON 2004, Santa Clara, CA, Oct. 2004, pp. 16-24.

10. A.-C. Pang and H.-W. Tseng, "Dynamic backoff for wireless personal networks," in IEEE GLOBECOM 2004, vol. 3, Dallas, TX, Dec. 2004, pp. 1580-1584.

11. M. Neugebauer, J. Plonnigs, and K. Kabitzsch, "A new beacon order adaptation algorithm for IEEE 802.15.4 networks," in Proceedings of the Second European Workshop on Wireless Sensor Networks, Istanbul, Turkey, Feb. 2005, pp. 302-311.

12. J. Mišić, S. Shafi, and V. B. Mišić, "The impact of MAC parameters on the performance of 802.15.4 PAN," Ad hoc Networks, vol. 3, no. 5, pp. 509-528, Sept. 2005.

13. - "Performance of beacon enabled ieee 802.15.4 cluster with downlink and uplink traffic," IEEE Transactions on Parallel and Distributed Systems, vol. 17, no. 4, pp. 361-377, Apr. 2006.

14. V. P. Mhatre, C. Rosenberg, D. Kofman, R. Mazumdar, and N. Shroff, "A minimum cost heterogeneous sensor network with a lifetime constraint," IEEE Transactions on Mobile Computing, vol. 4, no. 1, pp. 4-15, Jan-Feb 2005.

15. M. Yarvis, N. Kushalnagar, H. Singh, A. Rangarajan, Y. Liu, and S. Singh, "Exploiting heterogeneity in sensor networks," in Proc. INFOCOM05, vol. 2, Miami, FL, Mar. 2005, pp. $878-890$.

16. RSoft Design, Inc., Arifex v.4.4.2, San Jose, CA, 2003. 